\title{
ELTs and the study of individual, massive stars typical of "primordial" galaxies
}

\author{
Alexei A. Kniazev ${ }^{1}$ and Simon A. Pustilnik ${ }^{2}$ \\ ${ }^{1}$ South-African Astronomical Observatory, Cape Town, South Africa; \\ email: akniazev@saao.ac.za \\ ${ }^{2}$ Special Astrophysical Observatory RAS, Nizhnij Arkhyz, 369167 Russia \\ email: sap@sao.ru
}

\begin{abstract}
We discuss the opportunities to use the future ELTs to study in detail the properties and the evolution peculiarities of individual massive stars with a metal content typical of galaxies in the Universe during the first $0.5-1 \mathrm{Gyr}$, corresponding to the earliest known newly formed galaxies at redshifts of $z=6-10$. This is possible in principle due to the existence in the local Universe of starbursting galaxies with metallicities of $\sim 1 / 30 \mathrm{Z}_{\odot}$. The nearest such galaxies are DDO 68 at $\sim 6.5 \mathrm{Mpc}$ with $12+\log (\mathrm{O} / \mathrm{H})=7.21$ and $\mathrm{I} \mathrm{Zw} 18$ at $15 \mathrm{Mpc}$ with $12+\log (\mathrm{O} / \mathrm{H})=7.17$. For the youngest star clusters (with ages of $\mathrm{T}<4-5 \mathrm{Myr}$ ) in these most metal-poor galaxies, stars with masses up to $40-60 \mathrm{M}_{\odot}$ should be present on both the Main Sequence and the later evolution stages, including the WR stage. They are expected to have apparent magnitudes as bright as $V=21^{m}$ for DDO 68 and $V=23^{m}$ for I Zw 18. Good S/Nratio spectroscopy with telescopes like OWL or JWST, allowing near-milli-arcsecond angular resolution, will provide unique information to check the most up-to-date models of massive star evolution in the very low metallicity regime, and thus, establish a firm basis to model the effects of star formation in 'primordial' galaxies, at the epoch of galaxy formation. Such observations will also provide an independent channel to probe the primordial Helium abundance.
\end{abstract}

Keywords. Stars: general, abundances, evolution, fundamental parameters; Galaxies: evolution.

\section{Introduction}

Massive stars of very low metallicity $\left(\lesssim 1 / 30 \mathrm{Z}_{\odot}\right)$ are the main energy and metal suppliers in young galaxies in the high-redshift Universe. However, our understanding of their structure and evolution, rotation, convection, element production and dispersal are rather scarce. Theoretical models of massive stars with a metallicity of $z \lesssim 0.0004$ currently can be checked only indirectly, through observations of HII regions around young starbursts in the most metal-poor local galaxies, and sometimes from the study of relative numbers of $\mathrm{WR}$ and $\mathrm{O}$ stars in these objects.

\section{The nearest extremely metal-poor galaxies}

The nearest known starbursting galaxies with $\mathrm{Z} \sim 1 / 30 Z_{\odot}$ and with many massive stars in both Main Sequence (MS) and post-MS stages are DDO 68 (D 6.5 Mpc, distance modulus $(\mathrm{dm})=29,12+\log (\mathrm{O} / \mathrm{H})=7.21)($ Pustilnik et al. 2005) and the well known I Zw $18(\mathrm{D} \sim 15 \mathrm{Mpc}, \mathrm{dm}=31,12+\log (\mathrm{O} / \mathrm{H})=7.17)$ (e.g., Izotov \& Thuan 2004). The low surface brightness dwarf UGCA 292 (D $~ 3 \mathrm{Mpc}, \mathrm{dm}=27.5,12+\log (\mathrm{O} / \mathrm{H})$ $=7.30$, van Zee 2000) also has two young star forming (SF) regions, albeit of low total $\operatorname{mass}\left(\sim 500 \mathrm{M}_{\odot}\right)$. 
For I Zw 18 there are very deep HST $V$ and $I$-band images, reaching $V=I=29$ th magnitude, which is equivalent to $\mathrm{M}_{V}=-2$. Thousands of stars are seen on the HST images above this limit. Hundred(s) among the brightest of them (with $V=24-27$ ) represent the massive star tail at various stages of their evolution.

For DDO 68 the recent SF episodes are localized mainly at its northern edge, in several compact regions along the ring-like filament. The ages of these regions vary in the range of 3 to $22 \mathrm{Myr}$, with total masses of young clusters up to $\sim 6 \times 10^{4} \mathrm{M}_{\odot}$.

\section{Spectroscopy of the most metal-poor massive stars with ELTs}

An ambitious task for future ELTs is to get good quality spectroscopy of these unique massive stars in the local Universe, as they represent also typical stars from the young very metal-poor galaxies at redshifts of 5-7. This gives an opportunity to study their rotation velocities, gravities, winds, abundance patterns. These very metal-poor massive stars provide also an alternative approach to the problem of primordial helium. Probably B-stars are more suitable due to the smaller effect of winds. While they are on the Main Sequence, $\mathrm{He} / \mathrm{H}$ in their atmosphere should be close to that of the gas from which the star formed.

Using the first results from VLT/UVES spectra of individual A-supergiants $(V=19.5$, $\mathrm{R}=20000, \mathrm{SNR}=30$, exposure time $=12.5 \mathrm{~h})$ in the nearby $(\mathrm{D}=1.32 \mathrm{Mpc}, \mathrm{dm}=25.6$ mag) dwarf irregular Sextans A (Kaufer et al. 2004), one can roughly estimate the requirements for ELT stellar spectroscopy of the nearest accessible, massive extremely metal-poor stars. O8-B0 stars with $\mathrm{M}_{V}=-(4-5)$ at the distance of DDO 68, with an apparent V magnitude of 24-25, will be well accessible with the OWL 100-m telescope, based on preliminary estimates, namely a Field of View of $1^{\prime} \times 1^{\prime}$, a spatial resolution of $0.02^{\prime \prime}$ and a spectral resolution of 1000-10000 (see Hook, 2005, and also http://www-astro.physics.ox.ac.uk/ imh/ELT). Having with OWL a gain in sensitivity relative to the VLT of $5.5 \mathrm{mag}$, and a spatial resolution of $0.6 \mathrm{pc}$, these massive stars will be well reachable for detailed studies with exposure times of several hours.

\section{References}

Hook, I.M. (ed.) 2005, "The Science Case for the European 50-100m telescope", OPTICON. See http://www.astro-opticon.org/networking/elt.html

Izotov, Y.I. \& Thuan, T.X. 2004, ApJ 616, 768

Kaufer, A., Venn, K.A., Tolstoy, E., Pinte, C. \& Kudritzki, R.-P. 2004, AJ 127, 2723

Pustilnik, S.A., Kniazev, A.Y. \& Pramskij, A.G. 2005, A\&A 443, 91

van Zee, L. 2000, ApJL 543, L31 